\title{
HEART FAILURE WITH MID-RANGE LEFT VENTRICULAR EJECTION FRACTION
}

DOI: 10.36740/WLek202008133

\author{
Olga A. Yepanchintseva', Oleg J. Zharinov², Kyrylo 0. Mikhaliev ${ }^{3}$ \\ 'HEART INSTITUTE OF THE MINISTRY OF HEALTH OF UKRAINE, KYIV, UKRAINE \\ 2SHUPYK NATIONAL MEDICAL ACADEMY OF POSTGRADUATE EDUCATION, KYIV, UKRAINE \\ ¿STATE INSTITUTION OF SCIENCE «RESEARCH AND PRACTICAL CENTER OF PREVENTIVE AND CLINICAL MEDICINE» STATE ADMINISTRATIVE \\ DEPARTMENT, KYIV, UKRAINE
}

\begin{abstract}
The aim of the publication was to review available data on epidemiology, pathophysiological and clinical aspects of HFmrEF as a specific HF pattern.

Materials and methods: We carried out the analysis of the publications that appeared during last decade, related to the different aspects of HFmrEF. The literature search was conducted by use of Google Web Search and PubMed search engines by the following key words: heart failure, left ventricular ejection fraction, mid-range, as well as their combinations.

Conclusions: Patients with specific HF pattern «HFmrEF» demonstrate multidirectional dynamic of systolic heart function with the possibility of transition to the category of reduced or preserved LVEF. Such patients need to be evaluated individually. Their management is based on neurohumoral modulators in order to prevent further LV dysfunction progression and repeated decompensation of $\mathrm{HF}$.
\end{abstract}

KEY WORDS: heart failure, left ventricular ejection fraction, mid-range

Wiad Lek. 2020;73(8):1765-1770

\section{INTRODUCTION}

Left ventricular (LV) ejection fraction (EF) is a basic instrumental parameter to classify heart failure (HF), assess survival prognosis and manage patients. Current standards of HF treatment are primarily based on dividing patients into categories of reduced and preserved LVEF. Until recently the cutoff for this division has been $45 \%$ [1]. However, in numerous clinical trials on the treatment of HF patients with reduced LVEF the inclusion criterion was predominantly LVEF less than 35 or $40 \%$, which is the basis for the current guidelines on the use of several classes of drugs and devices. On the other hand, the study of HF with preserved LVEF included patients with more than $40 \%, 45 \%$, or $50 \%$. Considering this, the evidence base for the management of patients with mid-range LVEF is quite scarce. Initially the level of $40-49 \%$ was termed the «gray zone» [2], whereas in the recent guidelines of the European Cardiac Society for the Diagnosis and Treatment of HF it was defined as the «mid-range» LVEF [3].

According to European guidelines the appropriate diagnosis of HF with mid-range LVEF requires compliance with the following conditions: 1) symptoms and / or signs of HF; 2) LVEF 40-49\%; 3) the level of B-natriuretic peptide $\geq 35 \mathrm{pg}$ / ml or NT-pro-B-natriuretic peptide $\geq 125 \mathrm{pg} / \mathrm{ml}$; 4) echocardiographic signs of structural heart disease: left ventricular hypertrophy - myocardial mass index $>115 \mathrm{~g} / \mathrm{m}^{2}$ in men and $>95 \mathrm{~g} / \mathrm{m}^{2}$ in women, left atrial dilatation volume index $\geq 34 \mathrm{ml} / \mathrm{m}^{2}$, or $\mathrm{LV}$ diastolic dysfunction - E / e' $\geq 13$ and average velocity e ' $<9 \mathrm{~cm} / \mathrm{s}$ ). The authors of recent European guidelines have drawn attention to the heterogeneity of clinical manifestations, treatment and prognosis of HF with mid-range LVEF [3].

It is important to identify the category of patients with "midrange" LVEF values because this "gray zone" may be a stage of either improving or restoring LV systolic function, or its worsening [4]. According to the authors of Spanish observational study REDINSCOR II conducted on in-hospital patients with HF, «legitimizing» a new subgroup of patients with HF does not fundamentally change the existing approaches to risk stratification [5]. However, it provides a basis for further study and analysis of the features of development, diagnosis, and treatment of HF in patients with mid-range levels of LVEF. In which cases can the recognized approaches to the treatement of patients with reduced LVEF be extended to the patients of the mid-range category still remains a most important question. Answer to this question may change the ideology of using several classes of drugs and devices not only in terms of treatment, but also prevention of the progression of LV dysfunction and HF.

\section{THE AIM}

The aim of the publication was to review available data on epidemiology, pathophysiological and clinical aspects of $\mathrm{HF}$ in patients with mid-range LVEF as a specific HF pattern.

\section{MATERIALS AND METODS}

We carried out the analysis of the publications that appeared during last decade, related to the different aspects 
of HF with mid-range LVEF. While performing literature search, we took into consideration the results of certain landmark studies, published earlier than the pre-specified search period. The literature search was conducted by use of Google Web Search and PubMed search engines by the following keywords: heart failure, left ventricular ejection fraction, mid-range, as well as their combinations.

\section{REVIEW AND DISCUSSION}

\section{ETIOLOGICAL FACTORS}

HF can be caused by virtually any myocardial lesion, and determining the cause of the disease is crucial for its effective treatment. For example, among the patients with reduced LVEF prevail those with postinfarction cardiosclerosis, dilated cardiomyopathy, as well as diabetes mellitus and other cardiovascular risk factors [6]. On the other hand, HF with preserved LVEF is more likely to occur in elderly patients, women, and in hypertension [7]. Apparently, in a large part of patients the reduction of LVEF to its mid-range is a stage of progression of LV dysfunction after the onset of clinical manifestations of $\mathrm{CAD}$, in particular. It is no coincidence that in one of the first publications this category was named "middle child" among patients with HF [8]. In various registry studies the proportion of patients with mid-range LVEF was between 13 and $24 \%$ of all HF patients [9-12]. In the GWTG-HF registry including over 40,000 in-hospital patients from 2005 to 2010 with both a downward tendency in proportion of HF patients with reduced LVEF and an upward tendency in proportion of HF patinets with preserved LVEF, the proportion of HF patients with mid-range LVEF was virtually unchanged [13].

The development of HF with mid-range LVEF may be associated with diastolic and mild systolic dysfunction of the LV. In large OPTIMIZE-HF and ADHERE registry trials this category occupied an intermediate position between groups of patients with preserved and reduced LVEF $[14,15]$ by many clinical characteristics, prognosis, and treatment features. It should be emphasized that concomitant diseases such as atrial fibrillation, chronic obstructive pulmonary disease, anemia, and kidney failure are often present in patients with mid-range LVEF [11]. In comparison with the category of reduced LVEF they are more likely to have higher blood pressure, whereas myocardial ischemia and diabetes occur more often compared to the patients with preserved LVEF [8].

\section{MECHANISMS OF DEVELOPMENT}

The number of specific studies on the pathophysiology of HF with mid-range LVEF is still limited. It is obvious that by the level of the brain natriuretic peptide and troponins the indicated category of patients occupies an intermediate place between the categories of reduced and preserved LVEF, while increasing level of biomarkers along with the progression of LV dysfunction is associated with the stretching of myocardial fibers [16]. The dynamics of the level of biomarkers, in particular, NT-pro-brain natriuretic peptide, may be a sensitive indicator of the survival prognosis of HF patients, including those with mid-range LVEF $[17,18]$.

Probably, variability of the LVEF index and its possible transition from one category to another is most important in determining specific aspects of patient's management and predicting the course of the disease. First of all, it is logical to assume that the reduction of LVEF to the mid-range may be a stage in progression of left ventricular dysfunction and HF, with most patients initially developing HF with preserved LVEF. However, apart from the range of LVEF levels, there are no specific features that would distinguish the intermediate LVEF group from two other categories of patients with HF $[19,20]$. In the GWTG-HF registry this category was similar to both "extreme" groups of patients (with reduced and preserved HF) by demographic characteristics, comorbidities, laboratory parameters, and frequency of neurohumoral modulators use, yet it was more similar to the cohort of patients with preserved LVEF [9]. However, CAD is more likely to be present in mid-range LVEF patients with HF than in patients with preserved LVEF. Moreover, the addition of ischemia may cause transition of patients from the category of preserved LVEF to the mid-range [21, 22]. Conversly, our findings indicate that myocardial revascularization make possible the transition of one third of patients from the mid-range to the preserved LVEF category [23]. In general, the results of registry studies show that basing risk stratification of adverse events in patients with HF solely on LVEF index has significant limitations $[5,21]$.

LVEF transition to the mid- range may also be a consequence of its decrease due to the reverse factors. For example, in patients with atrial fibrillation, LVEF is often reduced during an arrhythmic episodes, but may recover after a sinus rhythm is restored. It is well known that in most patients with both atrial fibrillation and HF arrhythmia is a main driving force of the onset or progression of HF [24]. On the other hand, in the presence of adequate management of a patient with HF and reduced LVEF an increase in LVEF can be often observed. This parameter reaches the "plateau" (in many cases it is in the mid-range) and can remain relatively stable over a long period of time in patients compliant to the necessary treatment $[25,26]$. Conditions in which the likelihood of LV reverse remodeling is particularly high are tachycardiomyopathy, peripartum cardiomyopathy, thyrotoxicosis, active myocarditis, resistant arterial hypertension, left bundle branch block, effect of cardiotoxic chemotherapy [27]. Prescription of rational therapy in such patients makes possible the restoration of LVEF and their transition to the group of the preserved LVEF.

Thus, the ways for a patient to «fall» into the category of mid-range LVEF might vary: 1 ) as a stage of heart disease progression; 2) potentially reverse moderate decrease in LVEF; 3) partially restored LV function in patients with decreased LVEF baseline (Fig. 1).

According to the University of Washington HF registry, $73 \%$ of patients with mid-range LVEF demonstrated 


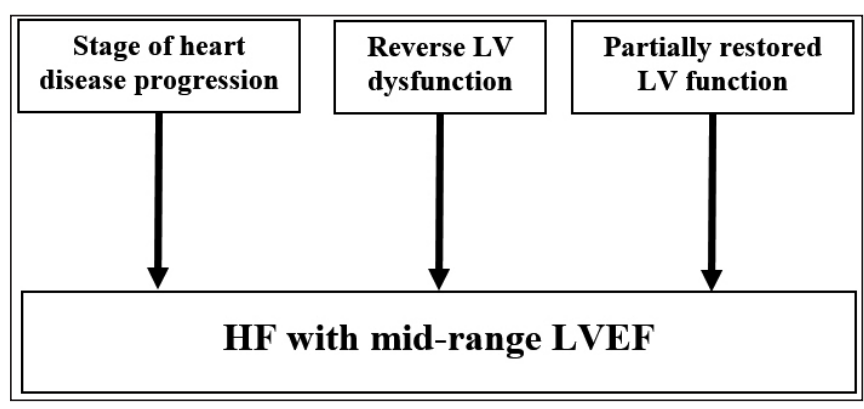

Fig. 1. Development of HF with mid-range LVEF.

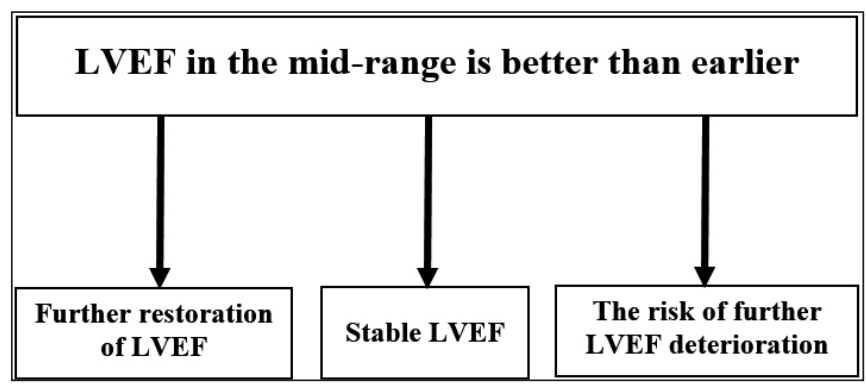

Fig. 2. Possible options of LVEF dynamics in patients with better mid-range LVEF than before.

reduced LVEF in preliminary studies, $17 \%$-preserved LVEF, and $10 \%$ remained in the same LVEF category as before [21]. Hence, the group of mid-range patients was quite heterogeneous, with the majority of those with the "restored" LVEF; whereas, there were relatively few cases of LVEF "worsening" to the mid-range. This population of patients, in its turn, may prove the importance of assessing the previous course of the disease in determining treatment strategy, and in many cases, may serve a benchmark for maintaining the achieved LVEF "restoration" level.

It should be emphasized that evaluation of LVEF dynamics against the background of treatment of heart disease should take into account limitations of the research methods used. It is important to correctly assess LVEF according to the existing standards, in particular, taking into account the presence of mitral regurgitation [28]. Inaccurate measurement of EF may result in non-compliance with therapeutic standards, as well as incorrect assessment of patients' condition in dynamics. In particular, in a cohort of 2032 patients with ischemic HF and reduced LVEF who participated in the STICH study, comparisons were made of LVEF findings obtained by echocardiography, single-photon emission computed tomography, and magnetic resonance imaging [29]. It turned out that in more than a half of the evaluated patients discrepancies in LVEF measured by different methods exceeded $5 \%$. Therefore, the authors suggest that estimating LVEF in dynamics should rely only on one method of LVEF measuring.

PREVENTION OF HEART FAILURE PROGRESSION There are no specific recommendations in the European guidelines for the management of HF patients with mid- range LVEF. However, since these patients were predominantly enrolled in the treatment of HF with preserved LVEF, experts suggest that the principles of their treatment should be similar to those for patients with preserved LVEF prior to obtaining the appropriate evidence base [3]. On the other hand, the choice of therapeutic agents should be made from the standpoint of prevention of HF progression and blood circulation decompensation caused by transition of patients to the category of reduced LVEF. Obviously, the basis for treatment should be drugs usually recommended for patients with reduced LVEF, namely renin-angiotensin system blockers, beta-blockers and antagonists of mineralocorticoid receptors. Moreover, in many cases, given the severity of decompensation, the presence of resistant arterial hypertension, concomitant atrial fibrillation, and other HF progression predictors, the therapy with neurohumoral modulators may be initiated prior to the reduction of LVEF to critical level - less than $40 \%$ or $35 \%$. It is logical to assume that preventive use of drugs proven to be effective in HF patients with reduced LVEF may in many cases modify the course of the disease and slow HF progression rate in patients with mid-range LVEF. It is of particular importance to evaluate causes of $\mathrm{HF}$ and to consider its interventional treatment in the presence of CAD. The analysis of individual clinical features may determine the feasibility of using devices in individual patients with mid-range LVEF.

Given the absence of specific evidence base for the management of mid-range LVEF patients, the main source of information so far have been retrospective analyses of data from previously conducted studies. In particular, in the CHARM-Preserved study candesartan reduced the risk of hospitalizations due to HF in patients with mid-range LVEF similar to the category of patients with reduced LVEF [30]. Analysis of the results of the TOPCAT study showed that spironolactone was effective at LVEF levels of $45-49 \%$ [31]. In a meta-analysis of 11 clinical studies in patients with mid-range LVEF, the effect of beta-blockers was similar to that with reduced LVEF. Moreover, on the background of treatment an increase in LVEF and a favorable effect on the prognosis for patients' survival was observed [32]. Apparently, these retrospective data may provide a basis for further prospective randomized trials.

A promising direction in the evolution of modern HF treatment is the use of biomarkers to evaluate treatment effectiveness. In a TIME-CHF study, treatment based on NT-pro-B-natriuretic peptide level assessment in dynamics allowed to reduce the risk of hospital admission due to HF in patients with mid-range LVEF, similar to HF with reduced LVEF [33]. At the same time, treatment with sacubitril / valsartan did not significantly lower rate of total hospitalizations for HF and death from cardiovascular causes among patients with HF and LVEF of 45\% or higher, compared to valsartan, in recently completed PARAGON-HF (NCT01920711) study [34]. Yet, further analyses will help to determine which patients with $\mathrm{HF}$ and mid-range or preserved LVEF might benefit most from sacubitril / valsartan. 
Compared to HF with reduced LVEF, patients with mid-range LVEF are less likely to receive diuretics, beta-blockers, mineralocorticoid receptor antagonists, angiotensin-converting enzyme inhibitors and sartans $[3,35]$. Experts of the American Heart Association recommend to consider the appropriateness of prescribing mineralocorticoid receptor antagonists to HF patients and those with LVEF $>45 \%$ in order to reduce the number of hospital admissions and diuretics - to eliminate congestive manifestations [36]. At the same time, one should perform the search and correction of background cardiovascular diseases, more specifically, CAD and uncontrolled arterial hypertension, as well as other concomitant pathologies: DM, chronic kidney disease, chronic obstructive pulmonary diseases. Their treatment is an important component of HF treatment with mid-range LVEF, similar to HF with preserved LVEF [10,37].

\section{DYNAMICS OF EJECTION FRACTION AND TREATMENT DURATION}

LVEF changes are a sensitive indicator of disease progression or, conversely, a possible consequence of its effective treatment. The existing body of evidence allows to suggest that LVEF dynamics is more closely related to the survival of patients than LVEF baseline level. In particular, the transition of patients from mid-range category to that of reduced LVEF is associated with a worse prognosis than maintaining the baseline level or LVEF restoration to normal rates $[6,35]$. In the OPTIMIZE-HF study, mortality was $3.9 \%$ in patients with reduced LVEF, $3.0 \%$ in the midrange, and 2.9\% in HF patients with preserved LVEF [38]. According to the data of meta-analysis conducted in more than 40,000 HF patients, the risk of death progressively increased with a decrease in LVEF for every $5-10 \%$ in the category of patients with LVEF below $40 \%$, but did not differ significantly in the subgroups of patients with LVEF 40 or more \% [6]. In contrast, chronic kidney disease has been a strong predictor of survival prognosis in patients with mid-range LVEF or with preserved LVEF [39].

In a recently published analysis of the Swedish registry data, all patients were divided into three groups depending on the LVEF index: $50 \%$ or more, $40-49 \%$, and less than $40 \%$ [40]. The study analyzed the dependence of LVEF dynamics on the background diseases and conditions such as hypertension, CAD, the possibility of LVEF changes and its effect on the number of hospital admissions and patient mortality. In the cohort of 886 patients there was a transition of patients with preserved LVEF to other categories over the course of the study: $25 \%$ demonstrated increased LVEF, 38\% transited to the "gray zone», 37\% - LVEF became less than $40 \%$. Ischemic heart disease was a predictor of unfavorable LVEF dynamics, and the favorable one was arterial hypertension. On the contrary, LVEF remained unchanged in $16 \%$ of 937 patients with mid-range LVEF, in $10 \%$ it increased to $50 \%$ or more, and in $75 \%$ decreased to less than $40 \%$. At the same time in patients with reduced and mid-range LVEF transition to the category of LVEF over $50 \%$ was associated with a lower risk of hospital admission and mortality.

Importantly, favorable LVEF dynamics allows one to hope for a better course of disease and survival prognosis for patient, as demonstrated in one of the retrospective cohort studies involving $2166 \mathrm{HF}$ outpatients [41]. At three-year follow-up LVEF improvement or recovery in HF patients was associated with lower mortality, fewer adverse cardiovascular events, and hospital admissions. This aspect is fully confirmed for the category of patients with mid-range LVEF: the course of disease and survival prognosis are better in the subcategory of «restored» LVEF (in patients who have previously demonsrated reduced LVEF) compared with those with no favorable LVEF dynamics [26].

Whether it is feasible to continue to take neurohumoral modulators after patient's transition to the category of mid-range or preserved LVEF when formal indicators for the use of these drugs disappear remains controversial. According to the Penn Heart Failure Study, patients whose LVEF has restored up to more than 50\% were similar in terms of their characteristics to HF patients with reduced LVEF, the category from which they have progressed to a «better» condition [42]. Despite improvements in LVEF, processes characteristic of neurohumoral activation, myocardial damage, and systemic inflammation persisted in a large share of patients. Therefore, patients with recovered LVEF remain at high risk of further LVEF reduction and HF decompensation [43]. In a recent study global longitudinal strain in patients with the so-called "HF with recovered LVEF " has made it possible to identify a group of individuals at an increased risk of recurrent LVEF reduction [44]. In this respect it has been suggested to characterize such patients as those with "HF with better LVEF than before" - rather than "with recovered LVEF" (if the level is $\geq 50 \%$ ) or "with improved LVEF" (40-49\%). There are two subpopulations in the mentioned category: patients with the risk of further deterioration ("myocardial remission"), as well as patients with stable recovery of functional status of the myocardium. In addition, in some patients it is possible to expect further increase of LVEF to normal level (Fig. 2).

Existing body of evidence suggests that in the event of transition from reduced to mid-range or mid-range to preserved LVEF there should be no rush in discontinuing drugs that helped a patient to overcome decompensation stage, since the risk of relapse in such patients increases sharply. This was confirmed by the pilot TRED-HF study [45] where patients with non-ischemic cardiomyopathy were randomized into two equal groups: one in which treatment was discontinued immediately after stabilization and the second one which continued the treatment for the next 6 months. The results of the study showed that the risk of relapse after discontinuation of treatment increases by $44 \%$, whereas no such case was recorded in the treatment group. Therefore, in any case, there is no need to speed up discontinuation of neurohumoral modulators, which helped to overcome the manifestations of decompensation 
and increase LVEF rate. Duration of intake of each of the used drugs should be determined individually.

\section{CONCLUSIONS}

The category of patients with mid-range LVEF (40-49\%) covers up to a quarter of all patients with $\mathrm{HF}$ and is characterized by heterogeneity of etiological factors and mechanisms of development. Such patients demonstrate multidirectional dynamic of systolic heart function with the possibility of transition to the category of reduced or preserved LVEF. LVEF changes are a more sensitive indicator of disease progression and prognosis than the baseline LVEF. Patients with midrange LVEF need to be evaluated individually for the use of neurohumoral modulators in order to prevent further LV dysfunction progression and repeated decompensation of HF.

\section{REFERENCES}

1. Dickstein K., Cohen-Solal A., Filippatos G. et al. ESC Guidelines for the diagnosis and treatment of acute and chronic heart failure 2008. Eur Heart J. 2008;29:2388-2442. doi: 10.1016/j.ejheart.2008.08.005.

2. McMurray J.J., Adamopoulos S., Anker S.D. et al. ESC Guidelines for the diagnosis and treatment of acute and chronic heart failure 2012: The Task Force for the Diagnosis and Treatment of Acute and Chronic Heart Failure 2012 of the European Society of Cardiology. Developed in collaboration with the Heart Failure Association (HFA) of the ESC. Eur Heart J. 2012;33:1787-1847. doi: 10.1093/eurheartj/ehs104.

3. Ponikowski P., Voors A.A., Anker S.D. et al. 2016 ESC Guidelines for the diagnosis and treatment of acute and chronic heart failure: The Task Force for the diagnosis and treatment of acute and chronic heart failure of the European Society of Cardiology (ESC). Eur Heart J. 2016;37:21292200. doi: 10.1093/eurhearti/ehw128.

4. Nadar S., Tariq 0 . What is heart failure with mid-range ejection fraction? A new subgroup of patients with heart failure. Card Fail Rev. 2018;4(1):6-8. doi: 10.15420/cfr.2018:7:2.

5. Gómez-Otero I., Ferrero-Gregori A., Varela Román A. et al. Mid-range ejection fraction does not permit risk stratification among patients hospitalized for heart failure. Rev Esp Cardiol. 2017;70:318-346. doi: 10.1016/j.rec.2016.11.016.

6. The survival of patients with heart failure with preserved or reduced left ventricular ejection fraction: an individual patient data meta-analysis. Eur Heart J. 2012;33:1750-1757. doi: 10.1093/eurheartj/ehr254.

7. Andersson C., Vasan R.S. Epidemiology of heart failure with preserved ejection fraction. Heart Fail Clin. 2014;10(3):377-388. doi: 10.1016/j. hfc.2014.04.003.

8. Lam C.S., Solomon S.D. The middle child in heart failure: heart failure with mid-range ejection fraction (40-50\%). Eur J Heart Fail. 2014;16:1049-1055. doi: 10.1002/ejhf.159.

9. Cheng R.K.,CoxM., Neely M.L. etal. Outcomes in patients with heartfailure with preserved, borderline, and reduced ejection fraction in the Medicare population.Am HeartJ.2014;168:721-730.doi:10.1016/j.ahj.2014.07.008.

10. Hsu J.J., Ziaeian B., Fonarow G.C. Heart Failure with mid-range (borderline) ejection fraction: clinical implications and future directions. JACC Heart Fail. 2017;5(11):763-771. doi: 10.1016/j.jchf.2017.06.013.

11. Kapoor J.R., Kapoor R., Ju C. et al. Precipitating clinical factors, heart failure characterization, and outcomes in patients hospitalized with heart failure with reduced, borderline, and preserved ejection fraction. JACC Heart Fail. 2016;4:464-472. doi: 10.1016/j.jchf.2016.02.017.
12. Tsuji K., Sakata Y., Nochioka K. et al. Characterization of heart failure patients with mid-range left ventricular ejection fraction-a report from the CHART-2 study. Eur J Heart Fail. 2017;19:1258-1269. doi: 10.1002/ ejhf.807.

13. Steinberg B.A., Zhao X., Heidenreich P.A. et al. Trends in patients hospitalized with heart failure and preserved left ventricular ejection fraction: prevalence, therapies, and outcomes. Circulation. 2012;126(1):65-75. doi: 10.1161/CIRCULATIONAHA.111.080770.

14. Fonarow G.C., Abraham W.T., Albert N.M. et al. Prospective evaluation of beta-blocker use at the time of hospital discharge as a heart failure performance measure: results from OPTIMIZE-HF. J Card Fail. 2007;13:722-731. doi: 10.1016/j.cardfail.2007.06.727.

15. Sweitzer N.K., Lopatin M., Yancy C.W. et al. Comparison of clinical features and outcomes of patients hospitalized with heart failure and normal ejection fraction ( $>$ or $=55 \%$ ) versus those with mildly reduced ( $40 \%$ to $55 \%)$ and moderately to severely reduced $(<40 \%)$ fractions. Am J Cardiol. 2008;101:1151-1156. doi: 10.1016/j. amjcard.2007.12.014.

16. Gohar A., Chong J.P.C., Liew 0.W. et al. The prognostic value of highly sensitive cardiac troponin assays for adverse events in men and women with stable heart failure and a preserved vs. reduced ejection fraction. Eur J Heart Fail 2017;19:1638-1647. doi: 10.1002/ejhf.911.

17. Albakri A. Heart failure with mid-range ejection fraction A review of clinical status and meta-analysis of clinical management methods. Trends in Res. 2018;1(4): 1-2. doi: 10.15761/TR.1000121.

18. Savarese G., Hage C., Orsini N. et al. Reductions in N-terminal probrain natriuretic peptide levels are associated with lower mortality and heart failure hospitalization rates in patients with heart failure with mid-range and preserved ejection fraction. Circ Heart Fail. 2016;9(11):e003105. doi: 10.1161/CIRCHEARTFAILURE.116.003105.

19. Chioncel O., Lainscak M., Seferovic P.M. et al. Epidemiology and oneyear outcomes in patients with chronic heart failure and preserved, mid-range and reduced ejection fraction: an analysis of the ESC Heart Failure Long-Term Registry. Eur J Heart Fail. 2017;19:1574-1585. doi: 10.1002/ejhf.813.

20. Pascual-Figal D.A., Ferrero-Gregori A., Gomez-Otero I. et al. Midrange left ventricular ejection fraction: clinical profile and cause of death in ambulatory patients with chronic heart failure. Int J Cardiol. 2017;240:265-270. doi: 10.1016/j.jicard.2017.03.032.

21. Rastogi A., NovakE., Platts A.E. etal. Epidemiology, pathophysiology and clinical outcomes for heart failure patients with a mid-range ejection fraction. Eur J Heart Fail. 2017;19(12): 1597-1605. doi: 10.1002/ ejhf.879.

22. Vedin 0., Lam C., Koh A. et al. Significance of ischemic heart disease in patients with heart failure and preserved, midrange, and reduced ejection fraction: a nationwide cohort study. Circ Heart Fail. 2017;10(6)::003875. doi: 10.1161/CIRCHEARTFAILURE.117.003875.

23. Yepanchintseva 0.A., Zharinov 0.J., Mikhaliev K.O. et al. Dysfunktsiia livoho shlunochka pered planovoiu khirurhichnoiu revaskuliaryzatsiieiu. Osoblyvosti «siroi zony» [Left ventricular dysfunction before planned surgical revascularization. Features of the "grey area»]. Ukrainian Journal of Cardiology. 2018;1:29-44. (In Ukrainian).

24. Smit M.D., Moes M.L., Maass A.H. et al. The importance of whether atrial fibrillation or heart failure develops first. Eur J Heart Fail. 2012;14(9):1030-1040. doi: 10.1093/eurjhf/hfs097.

25. Lupón J., Gavidia-Bovadilla G., Ferrer E. et al. Dynamic trajectories of left ventricular ejection fraction in heart failure. JACC. 2018;72(6):591-601. doi: 10.1016/j.jacc.2018.05.042. 
26. Nadruz W. Jr., West E., Santos M. et al. Heart failure and midrange ejection fraction: implications of recovered ejection fraction for exercise tolerance and outcomes. Circ Heart Fail. 2016;9(4):e002826. doi: 10.1161/CIRCHEARTFAILURE.115.002826.

27. Merlo M., Caiffa T., Gobbo M. et al. Reverse remodeling in dilated cardiomyopathy: insights and future perspectives. Int J Cardiol Heart Vasc. 2018;18:52-57. doi: 10.1016/j.jicha.2018.02.005.

28. Lang R.M., Badano L.P., Mor-Avi V. et al. Recommendations for cardiac chamber quantification by echocardiography in adults: an update from the American Society of Echocardiography and the European Association of Cardiovascular Imaging. J Am Soc Echocardiogr 2015;28:1-39.e14. doi: 10.1016/j.echo.2014.10.003.

29. Pellikka P.A., She L., Holly T.A. et al. Variability in ejection fraction measured by echocardiography, gated single-photon emission computed tomography, and cardiac magnetic resonance in patients with coronary artery disease and left ventricular dysfunction. JAMA Netw Open. 2018;1(4):e181456. doi:10.1001/jamanetworkopen.2018.1456.

30. Lund L.H., Claggett B., Liu J. Heart failure with mid-range ejection fraction in CHARM: characteristics, outcomes and effect of candesartan across the entire ejection fraction spectrum. Eur J Heart Fail. 2018;20(8):1230-1239. doi: 10.1002/ejhf.1149.

31. Solomon S.D., Claggett B., Lewis E.F. et al. Influence of ejection fraction on outcomes and efficacy of spironolactone in patients with heart failure with preserved ejection fraction. Eur Heart J. 2016;37:455-462. doi: 10.1093/eurheartj/ehv464.

32. Cleland J.G.F., Bunting K.V., Flather M.D. et al. Beta-blockers in Heart Failure Collaborative Group. Beta-blockers for heart failure with reduced, mid-range, and preserved ejection fraction: an individual patient-level analysis of double-blind randomized trials. Eur Heart J. 2018;39:26-35. doi: 10.1093/eurheartj/ehx564.

33. Rickenbacher P., Kaufmann B.A., Maeder M.T. et al. Heart failure with mid-range ejection fraction: a distinct clinical entity? Insights from the Trial of Intensified Versus Standard Medical Therapy in Elderly Patients With Congestive Heart Failure (TIME-CHF). Eur J Heart Fail. 2017;19(12):1586-1596. doi: 10.1002/ejhf.798.

34. Solomon S.D., McMurray J.J.V., Anand I.S. et al. Angiotensin-neprilysin Inhibition in heart failure with preserved ejection fraction. N Engl J Med. 2019. doi: 10.1056/NEJMoa1908655.

35. Butler J., Fonarow G.C., Zile M.R. et al. Developing therapies for heart failure with preserved ejection fraction: current state and future directions. JACC Heart Fail. 2014;2:97-112. doi: 10.1016/j. jchf.2013.10.006.

36. Yancy C.W., JanuzziJ.L.Jr., Allen L.A. etal.2017 ACC expert consensus decision pathway for optimization of heart failure treatment: answers to 10 pivotal issues about heart failure with reduced ejection fraction: a report of the American College of Cardiology Task Force on Expert Consensus Decision Pathways. JACC. 2018;71:201-230. doi: 10.1016/j.jacc.2017.11.025.

37. Ather S., Chan W., Bozkurt B. et al. Impact of noncardiac comorbidities on morbidity and mortality in a predominantly male population with heart failure and preserved versus reduced ejection fraction. JACC. 2012;59:998-1005. doi: 10.1016/j.jacc.2011.11.040.

38. Abraham W.T., Fonarow G.C., Albert N.M. et al. Predictors of inhospital mortality in patients hospitalized for heart failure. JACC. 2008;52(5):347-356. doi: 10.1016/j.jacc.2008.04.028.

39. Löfman I., Szummer K., Dahlström U. et al. Associations with and prognostic impact of chronic kidney disease in heart failure with preserved, mid-range, and reduced ejection fraction. Eur J Heart Fail. 2017;19:1606-1614. doi: 10.1002/ejhf.821.
40. Savarese G., Vedin 0., D'Amario D. et al. Prevalence and prognostic implications of longitudinal ejection fraction change in heart failure. JACC Heart Fail. 2019;7(4):306-317. doi: 10.1016/j.jchf.2018.11.019.

41. Kalogeropoulos A.P., Fonarow G.C., GeorgiopoulouV. et al. Characteristics and outcomes of adult outpatients with heart failure and improved or recovered ejection fraction. JAMA Cardiol. 2016;1(5):510-518. doi: 10.1001/jamacardio.2016.1325.

42. Basuray A., French B., Ky B. et al. Heart failure with recovered ejection fraction: clinical description, biomarkers and outcomes. Circulation. 2014;129(23):2380-2387. doi: 10.1161/CIRCULATIONAHA.113.006855.

43. De Groote P., Fertin M., Duva Pentiah A. et al. Long-term functional and clinical follow-up of patients with heart failure with recovered left ventricular ejection fraction after $\beta$-blocker therapy. Circ Heart Fail. 2014;7(3):434-439. doi: 10.1161/CIRCHEARTFAILURE.113.000813.

44. Adamo L., Perry A., Novak E. et al. Abnormal global longitudinal strain predicts future deterioration of left ventricular function in heart failure patients with a recovered left ventricular ejection fraction. Circ Heart Fail. 2017;10(6). pii: e003788. doi: 10.1161/CIRCHEARTFAILURE.116.003788.

45. Halliday B.P., Wassall R., Lota A.S. et al. Withdrawal of pharmacological treatment for heart failure in patients with recovered dilated cardiomyopathy (TRED-HF): an open-label, pilot, randomised trial. Lancet. 2019;393(10166):61-73. doi: 10.1016/S0140-6736(18)32484-X.

The study was conducted as a fragment of the complex scientific project of the Department of Heart and Vascular $\mathrm{Pa}$ thology Diagnostics (Heart Institute of the Ministry of Health of Ukraine) "Scientific validation of efficacy of myocardial revascularization for patients with coronary artery disease" (state registration number 0116U008820; term: 2016-2018).

\section{ORCID and contributionship:}

Olga A. Yepanchintseva: 0000-0001-7054-1564 A,B,D

Oleg J. Zharinov: 0000-0002-4089-9757 A,E,F

Kyrylo O. Mikhaliev: 0000-0003-3759-6699 D,E

\section{Conflict of interest:}

The Authors declare no conflict of interest.

\section{CORRESPONDING AUTHOR}

\section{Olga A. Yepanchintseva}

Heart Institute of the Ministry of Health of Ukraine

5 A Bratyslavska str., 02660 Kyiv, Ukraine

tel.: +380679929152

e-mail: 0.epanoly@gmail.com

Received: 10.10 .2019

Accepted: 03.06.2020

A - Work concept and design, B - Data collection and analysis, C - Responsibility for statistical analysis, D-Writing the article, $\mathbf{E}$-Critical review, $\mathbf{F}-$ Final approval of the article 